\title{
Application of a new speed control strategy in Tank Gun Control System
}

\author{
Jing Zhou, a , Bin Liu ${ }^{2, b}$, Ju-Qiu Lin ${ }^{3, c}$, Qing Lin ${ }^{4, d}$, \\ Qian Liu ${ }^{5, e}$, Ju-Yan Ni ${ }^{6, f}$, Yan-Yong Wang ${ }^{7, g}$ \\ ${ }^{1}$ Huai Shu Ling 4 yuan, Fengtai District, Bei Jing, China \\ ${ }^{2}$ Huai Shu Ling 4 yuan, Fengtai District, Bei Jing, China \\ ${ }^{3}$ Huai Shu Ling 4 yuan, Fengtai District, Bei Jing, China \\ ${ }^{4}$ Huai Shu Ling 4 yuan, Fengtai District, Bei Jing, China \\ ${ }^{5}$ Huai Shu Ling 4 yuan, Fengtai District, Bei Jing, China \\ ${ }^{6}$ Huai Shu Ling 4 yuan, Fengtai District, Bei Jing, China \\ ${ }^{7}$ Huai Shu Ling 4 yuan, Fengtai District, Bei Jing, China

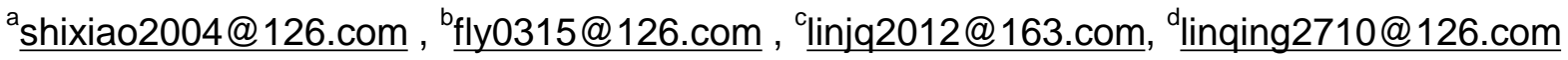 \\ Iq520502@sina.com, ${ }^{\mathrm{e}}$ jyni 2008@126.com, ${ }^{9}$ wangyanyongdl@126.com,
}

Keywords: Direct torque control, MRAS, SVPWM, full-order adaptive state observer.

Abstract. In order to make the tank armor vehicles lightweight, miniaturization, precision and high speed, a non-speed-sensor direct torque control strategy based on magnetic chain model reference adaptive system (MRAS) was designed, and the MRAS identification method based on the full-order adaptive state observer.Using Matlab/simulink simulation platform, a simulation model of the non-speed-sensor direct torque control based on the full-order adaptive state observer MRAS is buil, and the simulation results show that this new type of no-speed sensor control strategy is simple and effective, and is a method worthy of further study.

\section{Introduction}

The tank gun control system is an automatic control system that can drive the tank gun to the position determined by the fire control system timely. It can rapid firing, accurate targeting and artillery stabilization, and is the execution system on the main terminal of the tank fire control. Therefore, the tank gun control system is an important guarantee of the fire system performance.Three-phase ac permanent magnet synchronous motor with high efficiency density, high efficiency, high reliability and fast response ability have been applied to tank gun control system. The mathematical model of three-phase ac synchronous motor is the high order, nonlinear and strong coupling system with multi-variables.

The speed regulation strategy proposed in this paper is based on direct torque control. Direct torque control (DTC) is a kind of control method based on hexagon and even round flux trajectory that was proposed by Japanese scholar I.Takhashi and prof Depenbrock of Germany respectively in mid-eighties. This method solves the defects of vector control with the advantages of simplicity and easy realization of real-time control, such as the complexity of calculation and the influence of the characteristics of the motor parameters. At present, this technology has been successfully applied to the ac drive of tank gun control system.

In order to make the tank armor vehicles lightweight, miniaturization, precision and high speed, a non-speed-sensor direct torque control strategy based on magnetic chain model reference adaptive system (MRAS) was designed. On the one hand, this kind of control method eliminates the need for speed sensor installation and reduce the hardware investment, on the other hand, the application of permanent magnet synchronous motor in tank gun control system is more efficient for there is no obstacle caused by speed sensor to the development of speed control and position control. 


\section{The principle of direct torque control}

Mathematical model of permanent magnet synchronous motor in rotor coordinate system are as follows:

$$
\begin{aligned}
& {\left[\begin{array}{l}
u_{d} \\
u_{q}
\end{array}\right]=\left[\begin{array}{cc}
R+p L_{d} & -w L_{q} \\
w L_{d} & R+p L_{q}
\end{array}\right]\left[\begin{array}{l}
i_{d} \\
i_{q}
\end{array}\right]+\left[\begin{array}{c}
0 \\
w \psi_{f}
\end{array}\right]} \\
& \mathrm{t}_{e}=\frac{3 p_{n}\left|\psi_{s}\right|}{4 L_{\mathrm{d}} L_{q}}\left[2 \psi_{f} L_{q} \sin \delta_{s m}+\psi \psi_{s} \mid\left(L_{d}-L_{q}\right) \sin 2 \delta_{s m}\right]
\end{aligned}
$$

In the formula: $u_{d}, u_{q}, i_{d}, i_{q}, L_{d}, L_{q}$ are the components of voltage, current and inductance in $\mathrm{d}$ and

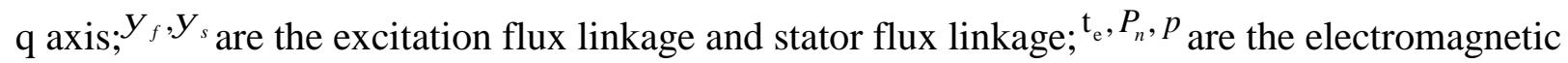
torque, the rotor pole pairs and the differential operator; $\delta_{\mathrm{sm}}$ is load angle.Formula (2) shows the core of the DTC is by controlling the load angle value to control the electromagnetic torque. The key problem the DTC technology needs to solve is how to keep the stator flux linkage amplitude constant in the process of motor running.

\section{The basic principle of SVPWM-DTC}

Space vector pulse width modulation (SVPWM), can also be called sinusoidal waveform of magnetic flux PWM. Its main idea is take the balanced three-phase motor stator magnetic chain ideal circular trajectory which powered by balanced three-phase sine wave voltage as a reference, to make the actual stator flux linkage vector close to the ideal circle track by switching inverter switch, then the high control performance is achieved.

Figure 1 shows the three-phase voltage type inverter circuit. To ensure that the voltage space vector flux trajectory is circular, combine the on-off state of power switch tube in the right order, adjust the switching time and improve the utilization of DC source voltage .

V1 V6 are six power switch tube. a、 b、 c are three bridge arm on-off state. When upper bridge arm is on state for " 1 ", otherwise for " 0 ", when lower bridge arm is on state for " 0 ",otherwise for "1".

In this way, a, b, c formed eight kinds of switch state : 000, 001, 010, 011, 100, 101, 110, 101. The eight kinds of switch state corresponding to eight basic voltage space vector, of which 000 and 111 voltage is zero, known as the zero state.For easy of use, with $U_{0} 、 U_{1} 、 U_{3} 、 U_{4} 、 U_{5} 、 U_{6} 、 U_{7}$ represent above eight kinds of switch state.

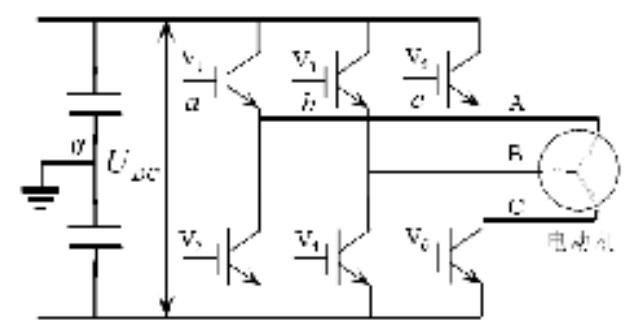

Figure 1 three-phase voltage type inverter circuit

Figure 2 shows the voltage space vector histogram. We use the method of SVPWM to optimize inverter switch state combination, so as to make the voltage vector provided by Inverter close to the desired ideal voltage vector. Specific methods is according to the sector location of desired voltage space vector, choose the adjacent non-zero voltage vector and zero voltage vector synthetic,to get the desired voltage space vector $u_{s}^{*}$ within the fixed sampling period. 


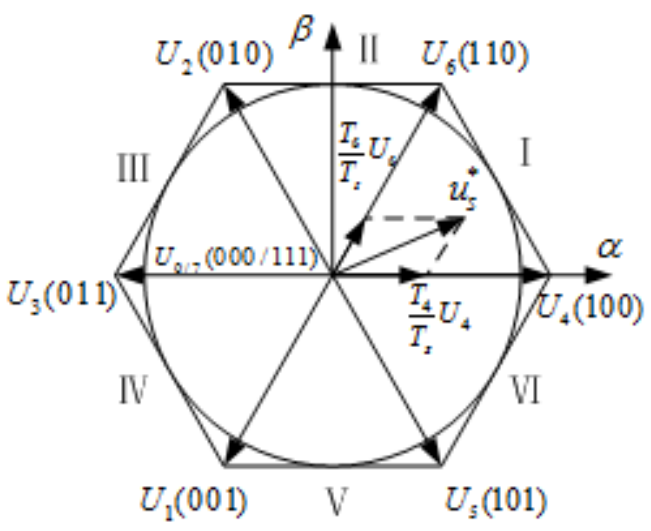

Figure 2 voltage space vector histogram

The key prolem of SVPM-DTC system is to get the voltage space vector that can compensate the deviation of stator flux and electromagnetic torque accuratly. In order to control the desired voltage space vector, we must determine the amplitude and phase of desired voltage vector. Here we use different mathematical models and methods to estimate the desired voltage space vector.

\section{(1) Generation of desired voltage space vector}

Forecasts thought is control of the stator flux and electromagnetic torque to track the expectations at the end of a sampling period, so as to control the change of the stator flux and electromagnetic torque in a sampling period.

When the amplitude and angle variable of stator flux linkage are known for $\left|\Psi_{\mathrm{s}} *\right|$ and $\Delta \theta$, the reference value $\left(\psi_{s c}\right)$ of the stator flux vector is given by formula (3).

$$
\psi_{s c}=\left|\psi_{s}^{*}\right| e^{j(\theta+\Delta \theta)}
$$

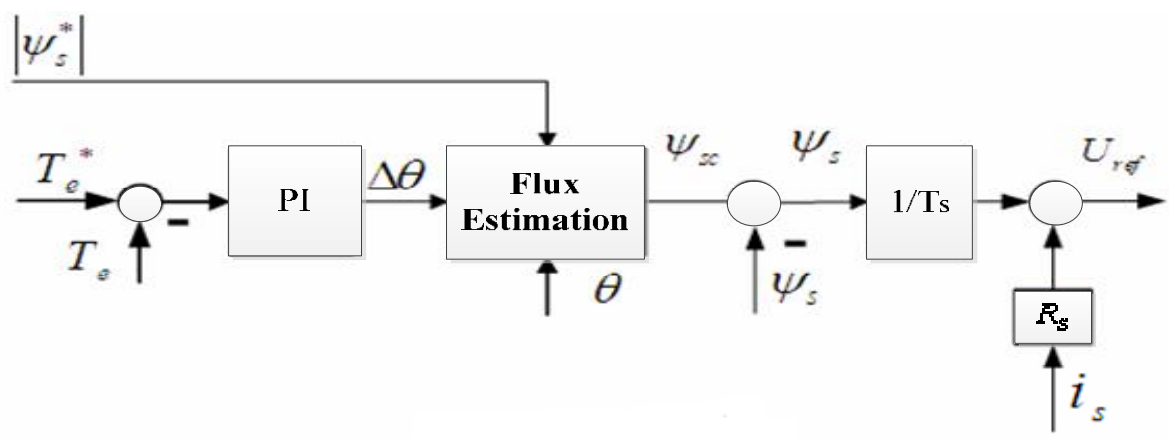

Figure 3 Generate desired voltage space vector

In formula (3), realized the closed-loop control of the magnetic chain amplitude by set the amplitude of the reference magnetic chain vector to the set value. In keeping the constant stator flux amplitude, under the condition of changing the phase angle of the stator flux linkage (the torque Angle) $\Delta \theta$, can change the value of the torque, and the $\Delta \theta$ can be got from PI adjustment the difference between given torque value and the observed torque value. As shown in formula (4) and (5):

$$
\begin{aligned}
\psi_{r}^{\mathrm{g}} & =\frac{R_{r}}{L_{\sigma}}\left(\psi_{s}-\psi_{r}\right)+j \omega \psi_{r} \\
\Delta \theta & =\left(k_{p}+\frac{k_{i}}{S}\right)\left(T_{e}^{*}-T_{e}\right)
\end{aligned}
$$

The expected voltage vector $U_{\text {ref }}$ is: 


$$
U_{r e f}=\frac{\Delta \psi_{s}}{T_{s}}+R_{s} i_{s}
$$

PS: $\Delta \psi_{s}=\psi_{s c}-\psi_{s}$

(2) The time of the expected voltage vector

First, the sector of the expected voltage vector should be judged. In two-phase stationary coordinate system, the expected voltage vector component is $u_{s \alpha}, u_{s \beta}$.

$$
\left\{\begin{array}{c}
u_{r e f 1}=u_{s \beta} \\
u_{r e f 2}=\left(\sqrt{3} u_{s \alpha}-u_{s \beta}\right) / 2 \\
u_{r e f 3}=\left(-\sqrt{3} u_{s \alpha}-u_{s \beta}\right) / 2
\end{array}\right.
$$

We define the following situations:

When $u_{\text {ref } 1}>0, \mathrm{~A}=1$, otherwise $\mathrm{A}=0$;

When $u_{\text {ref } 2}>0, \mathrm{~B}=1$, otherwise $\mathrm{B}=0$;

When $u_{\text {ref } 3}>0, \mathrm{C}=1$, otherwise $\mathrm{C}=0$;

Make $N=A+2 B+4 C$, the corresponding values of $N$ in six sectors are shown in table 1 .

Table 1 sector comparison table

\begin{tabular}{|l|l|l|l|l|l|l|}
\hline Sector & I & II & III & IV & V & VI \\
\hline$N$ & 3 & 1 & 5 & 4 & 6 & 2 \\
\hline
\end{tabular}

The Work order and action time of the switching voltage vector are determined by the sector. Only one switch state be change at a time to minimize the loss caused by switch tube when the expected voltage vector is synthesized. And, in order to reduce torque ripple, the expected voltage vector linear combination are established by two adjacent non-zero switching voltage vectors and zero voltage vectors. Take the example of figure 2 , when $u_{s}^{*}$ is in sector I, the adjacent non-zero voltage vectors $U_{4}, U_{6}$ and zero voltage vectors should be selected. The action time of the non-zero voltage vector and the zero vector as shown in formula (8).

In the two phases of the stationary coordinate system:

$$
\frac{2}{3} u_{d c}\left[\begin{array}{l}
1 \\
0
\end{array}\right] T_{4}+\frac{2}{3} u_{d c}\left[\begin{array}{c}
\cos 60^{\circ} \\
\sin 60^{\circ}
\end{array}\right] T_{6}=\left[\begin{array}{l}
u_{s \alpha} \\
u_{s \beta}
\end{array}\right] T_{s}
$$

PS: $T_{S}$ is sampling time, $u_{d c}$ is dc bus voltage.

According to the above derivation, we can get the action time of the expected voltage vector. Then combine the sector signal of $u_{s}^{*}$, the action time of two adjacent non-zero voltage vectors $T_{x}$ and $T_{y}$ are shown in table 2 .

$$
\begin{aligned}
& X=\sqrt{3} u_{s \beta} T_{s} / u_{d c} \\
& Y=\left(\frac{3}{2} u_{s \alpha}+\frac{\sqrt{3}}{2} u_{s \beta}\right) T_{s} / u_{d c} \\
& Z=\left(-\frac{3}{2} u_{s \alpha}+\frac{\sqrt{3}}{2} u_{s \beta}\right) T_{s} / u_{d c}
\end{aligned}
$$


Table 2. non-zero voltage vector action time

\begin{tabular}{|l|l|l|l|l|l|l|}
\hline Sector & I & II & III & IV & V & VI \\
\hline Tx & $-Z$ & Z & X & $-X$ & $-Y$ & Y \\
\hline Ty & X & Y & $-Y$ & Z & $-Z$ & $-X$ \\
\hline
\end{tabular}

In the practical application, especially in the case of motor speed mutation, as the increases of the motor torque ripple, the expected voltage vector may exceed the maximum output voltage of the inverter. Therefore, it is important to make overflow judgment on the time of switching vector. Determine whether $T_{x}+T_{y}>T_{s}$ is true. If not, keep $T_{x}$ and $T_{y}$ unchanged. Otherwise, the switching time of the device should be limited.And the time of two adjacent non-zero voltage vectors is $T_{x}^{*}$ and $T_{y}^{*}$.Their relationships with $T_{x}$ and $T_{y}$ are as follows:

$$
T_{x}^{*} / T_{x}=T_{y}^{*} / T_{y}
$$

The limited time of non-zero voltage vector can be obtained by the following formula:

$$
T_{x}^{*}=\frac{T_{x} \times T_{s}}{T_{x}+T_{y}}, T_{y}^{*}=\frac{T_{y} \times T_{s}}{T_{x}+T_{y}}
$$

In the practical application, due to the existence of the inverter dead zone time, if the action time of one voltage vector that amount three of switch voltage vector in the SWPWM over more than $70 \%$ of the control cycle, the other two voltage vector should be given up. Another situation is, if there is a very short time voltage vector, we give it up and divide it's action time to the other two voltage vectors equally because of the limitation of sampling period and dead band time.

Vector switching time is defined as follows:

$$
\begin{aligned}
& T_{a}=\left(T_{s}-T_{x}-T_{y}\right) / 4 \\
& T_{b}=T_{a}+T_{x} / 2 \\
& T_{c}=T_{b}+T_{y} / 2
\end{aligned}
$$

Then the switching schedule corresponding to three-phase of A, B and C in different sectors is shown in table 3.

Table 3 switch time

\begin{tabular}{|c|c|c|c|c|c|c|}
\hline Sector & I & II & III & IV & V & VI \\
\hline Tcm1 & $\mathrm{Ta}$ & $\mathrm{Tb}$ & $\mathrm{Tc}$ & $\mathrm{Tc}$ & $\mathrm{Tb}$ & $\mathrm{Ta}$ \\
\hline $\mathrm{Tcm} 2$ & $\mathrm{~Tb}$ & $\mathrm{Ta}$ & $\mathrm{Ta}$ & $\mathrm{Tb}$ & $\mathrm{Tc}$ & $\mathrm{Tc}$ \\
\hline $\mathrm{Tcm} 3$ & $\mathrm{Tc}$ & $\mathrm{Tc}$ & $\mathrm{Tb}$ & $\mathrm{Ta}$ & $\mathrm{Ta}$ & $\mathrm{Tb}$ \\
\hline
\end{tabular}

\section{Speed identification based on model reference system(MRAS)}

\section{Basic idea of model reference adaptive system}

In the coordinate system of $\alpha-\beta$, the current simulation model of magnetic chain (shown as formula (13) and formula (14)) contain the actual rotational speed information $\omega_{r}$, so the observation value of the rotor flux chain can be obtained as the adjustment model through the current simulation model; The voltage simulation model of magnetic chain (shown as formula (15) and formula (16)) do not contain the actual rotational speed information $\omega_{r}$, so the reference value of the rotor flux chain can be obtained as the reference model through the voltage simulation model.The physical relation between the rotor flux and the actual speed is simulated by PI regulator 
and the actual rotational speed can be calculated. The above is the basic idea of model reference adaptive method.

Current equation of rotor flux linkage under two phases of the stationary coordinate system are as follows:

$$
\begin{aligned}
& \Psi_{r \alpha}=\int\left(\frac{L_{m}}{T_{r}} i_{s \alpha}-\frac{\Psi_{r \alpha}}{T_{r}}-\omega_{r} \Psi_{r \beta}\right) d t \\
& \Psi_{r \beta}=\int\left(\frac{L_{m}}{T_{r}} i_{s \beta}-\frac{\Psi_{r \beta}}{T_{r}}+\omega_{r} \Psi_{r \alpha}\right) d t
\end{aligned}
$$

Voltage equation of rotor flux linkage under two phases of the stationary coordinate system are as follows:

$$
\begin{aligned}
& \psi_{r \alpha}=\frac{L_{r}}{L_{m}} \int\left[u_{s \alpha}-\left(R_{s}+\sigma L_{s} p\right) i_{s \alpha}\right] d t \\
& \psi_{r \beta}=\frac{L_{r}}{L_{m}} \int\left[u_{s \beta}-\left(R_{s}+\sigma L_{s} p\right) i_{s \beta}\right] d t
\end{aligned}
$$

The identification system block diagram of model reference adaptive is shown in figure 4:

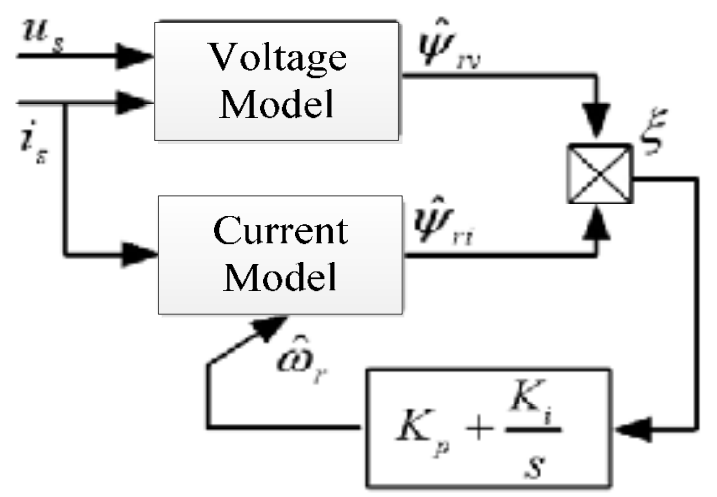

figure 4 The identification system block diagram of model reference adaptive PS, $\xi=\hat{\psi}_{r i \alpha} \hat{\psi}_{r v \beta}-\hat{\psi}_{r v \alpha} \hat{\psi}_{r i \beta}$

Then the speed identification equation is :

$$
\hat{\omega}_{r}=\left(K_{p}+\frac{K_{i}}{s}\right) \xi=\left(K_{p}+\frac{K_{i}}{s}\right)\left(\hat{\psi}_{r i \alpha} \hat{\psi}_{r i \beta}-\hat{\psi}_{r \alpha \alpha} \hat{\psi}_{r i \beta}\right)
$$

\section{Model reference adaptive (MRAS) speed identification based on the full-order adaptive state observer}

In the application, the MRAS system based on rotor flux estimation method is common. However, due to the existence of pure integral link, dc migration problem and the change of stator resistance, the identification accuracy of the conventional voltage model rotor flux linkage estimation method is low. Some improvement methods had been put forward by some scholars, such as to eliminate the influence of the pure integrator by adding the filter, as shown in figure 5 . But due to the change of stator resistance, the improvement is limited. 


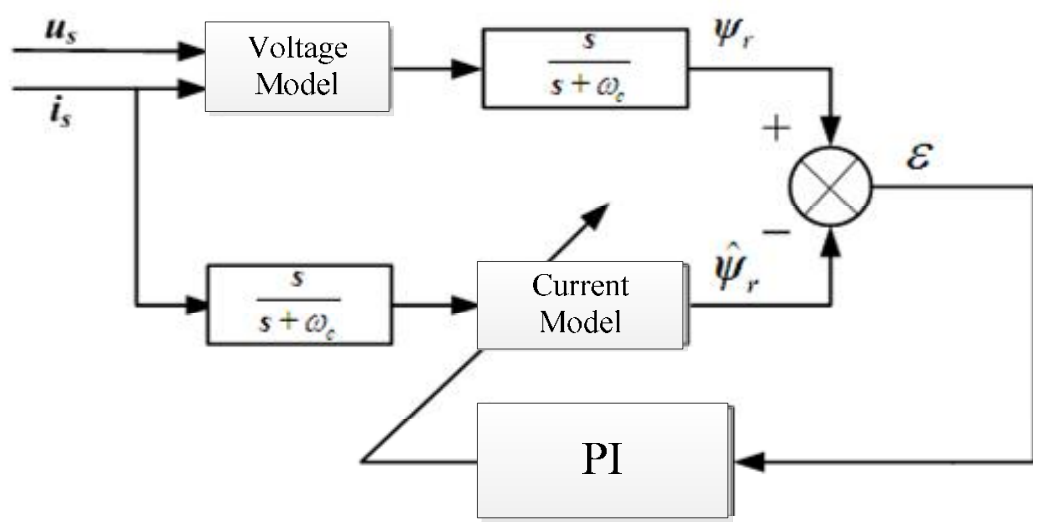

Figure.5 Improved rotor flux estimation method

Due to estimate speed and stator resistance at the same time is impossible,speed identification of the MRAS method mentioned before is not ideal. A MRAS identification method based on the full-order adaptive state observer is used in this paper.This method can recognize the speed and the stator resistance at the same time, so the precision of speed identification is improved. The structure is shown in figure 6.

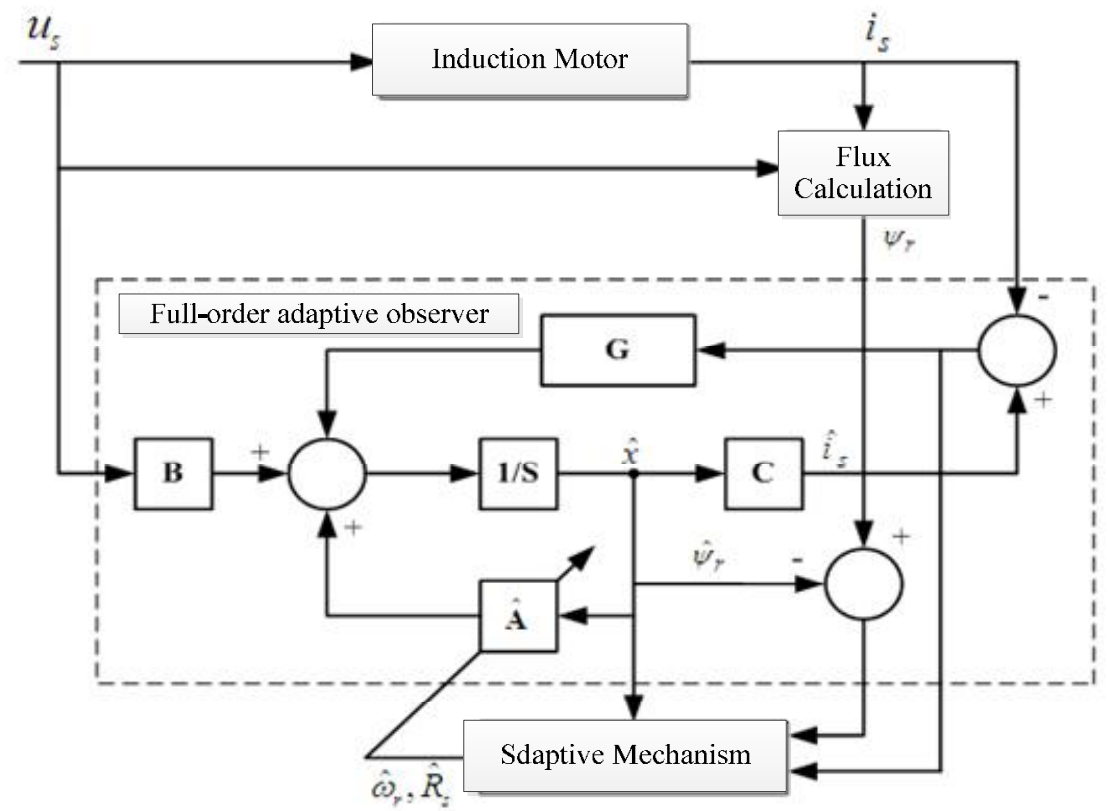

Figure 6. Identification of full-order MRAS

The mathematical model of induction motor is selected as the reference, and the whole order adaptive state observer that constructed according to the input stator voltage $u_{s}$ and output stator current $i_{s}$ is used as adjustable model and the adjustable parameter are $\omega_{r}, R_{S}$. If $\hat{i_{s}}=i_{s}$ is applicable to any input stator voltage, the adjustable parameters tend to be true, in the same way, the observation value of the rotor flux tends to be true $\hat{\psi}_{r}=\psi_{r}$.

Motor model:

$$
\begin{aligned}
& \delta=A x+B u_{s} \\
& y=C x \\
& \mathrm{PS}: \quad x=\left[\begin{array}{c}
i_{s} \\
\psi_{r}
\end{array}\right], \quad A=\left(\begin{array}{ll}
A_{11} & A_{12} \\
A_{21} & A_{22}
\end{array}\right), \quad B=\left[\frac{1}{\sigma L_{s}} I 0\right]^{T} \quad, \quad C=\left[\begin{array}{ll}
I & 0
\end{array}\right]^{T} \\
& A_{11}=-\left[\frac{R_{s}}{\sigma L_{s}}+\frac{1-\sigma}{\sigma T_{r}}\right] I, \quad A_{12}=-\frac{L_{m}}{\sigma L_{s} L_{r}}\left[\frac{1}{T_{r}} I-\omega_{r} J\right], \quad A_{21}=\frac{L_{m}}{T_{r}} I, \quad A_{22}=-\frac{1}{T_{r}} I+\omega_{r} J
\end{aligned}
$$


Full-order adaptive observer:

$$
\begin{aligned}
& \frac{d}{d t} \hat{x}=\hat{A} \hat{x}+B u_{s}+G\left(\hat{i}-i_{s}\right) \\
& \text { PS: } \hat{x}=\left[\begin{array}{c}
\hat{i}_{s} \\
\hat{\psi}_{r}
\end{array}\right], \quad \hat{A}=\left(\begin{array}{cc}
\hat{A}_{11} & \hat{A}_{12} \\
\hat{A}_{21} & \hat{A}_{22}
\end{array}\right), \\
& \hat{A}_{11}=-\left[\frac{\hat{R}_{s}}{\sigma L_{s}}+\frac{1-\sigma}{\sigma T_{r}}\right] I=a_{r 11} I, \quad \hat{A}_{12}=-\frac{L_{m}}{\sigma L_{s} L_{r}}\left[\frac{1}{T_{r}} I-\hat{\omega}_{r} J\right]=a_{r 12} I+a_{i 11} J \\
& \hat{A}_{21}=\frac{L_{m}}{T_{r}} I=a_{r 21} I, \quad \hat{A}_{22}=-\frac{1}{T_{r}} I+\hat{\omega}_{r} J=a_{r 22}+a_{i 22}
\end{aligned}
$$

The matrix feedback gain $\mathrm{G}$ is selected by the pole assignment, which is to use the observer pole to be proportional to the pole of the motor. We derive the adaptive law from the popov hyperstability theory, and take formula (18) minus formula (19):

$$
\begin{aligned}
& \frac{d}{d t} e=\frac{d}{d t}\left[\begin{array}{c}
e_{i_{s}} \\
e_{\psi_{r}}
\end{array}\right]=\frac{d}{d t}\left[\begin{array}{c}
i_{s} \\
\psi_{r}
\end{array}\right]-\frac{d}{d t}\left[\begin{array}{c}
\hat{i}_{s} \\
\hat{\psi}_{r}
\end{array}\right] \\
& =A x-\hat{A} \hat{x}-G\left(\hat{i}_{s}-i_{s}\right) \\
& =A(x-\hat{x}+\hat{x})-\hat{A} \hat{x}+G C e \\
& =(A+G C) e+\Delta A \hat{x} \\
& =(A+G C) e-W \\
& \text { PS: } e_{i_{s}}=\left[\begin{array}{c}
e_{i_{s \alpha}} \\
e_{i_{s \beta}}
\end{array}\right]=\left[\begin{array}{c}
i_{s \alpha}-\hat{i}_{s \alpha} \\
i_{s \beta}-\hat{i}_{s \beta}
\end{array}\right], \quad e_{\psi_{r}}=\left[\begin{array}{c}
e_{\psi_{r \alpha}} \\
e_{\psi_{r \beta}}
\end{array}\right]=\left[\begin{array}{l}
\psi_{r \alpha}-\hat{\psi}_{r \alpha} \\
\psi_{r \beta}-\hat{\psi}_{r \beta}
\end{array}\right] \\
& \Delta A=A-\hat{A} \\
& =\left[\begin{array}{cc}
0 & -\frac{-M}{\sigma L_{s} L_{r}} J \\
0 & J
\end{array}\right]\left(\omega_{r}-\hat{\omega}_{r}\right)+\left[\begin{array}{cc}
-\frac{1}{\sigma L_{s}} & 0 \\
0 & 0
\end{array}\right]\left(R_{s}-\hat{R}_{s}\right) \\
& =A_{\omega_{r}} \Delta \omega_{r}+A_{R_{s}} \Delta R_{s}
\end{aligned}
$$

$\mathrm{W}$ is a nonlinear time-varying channel, which is $W=-\Delta A\left[\begin{array}{c}\hat{i_{s}} \\ \hat{\psi_{r}}\end{array}\right]$

Based on the popov hyperstability theory, the condition for the system to be asymptotically stable is the transmission function of the system feedforward channel strictly positiv.

$$
\begin{aligned}
& \int_{0}^{t_{0}} e^{T} W d t \geq-\gamma^{2} \gamma=\text { const } \\
& k_{1}=\frac{M}{\sigma L_{s} L_{r}}, k_{2}=\frac{1}{\sigma L_{s}}, \text { then: } \\
& \int_{0}^{t_{0}} k\left\{\frac{d f(t)}{d t}\right\} f(t) d t=\frac{k}{2}\left\{f^{2}\left(t_{0}\right)-f^{2}(0)\right\} \geq-\frac{1}{2} k f^{2}(0)
\end{aligned}
$$

The adaptive law of the speed and stator resistance can be obtained: 


$$
\begin{aligned}
& \frac{d}{d t} \hat{\omega}_{r}=\left(\frac{e_{i_{s \beta}}}{k_{1}}+e_{\psi_{r \beta}}\right) \hat{\psi}_{r \alpha}-\left(\frac{e_{i_{s \alpha}}}{k_{1}}+e_{\psi_{r} \alpha}\right) \hat{\psi}_{r \beta} \\
& \frac{d}{d t} \hat{R}_{s}=-\frac{1}{k_{2}}\left(e_{i_{s \alpha}} i_{s \alpha}+e_{i_{s \beta}} i_{s \beta}\right)
\end{aligned}
$$

The proportional integral form is:

$$
\begin{aligned}
& \hat{\omega}_{r}=K_{p \omega}\left[e_{i_{s \beta}} \hat{\psi}_{r \alpha}-e_{i_{s \alpha}} \hat{\psi}_{r \beta}\right]+K_{i \omega} \int_{0}^{t}\left[e_{i_{s \beta}} \hat{\psi}_{r \alpha}-e_{i_{s \alpha}} \hat{\psi}_{r \beta}\right] d t \\
& \hat{R}_{s}=K_{p R_{s}}\left[-\left(e_{i_{s \alpha}} i_{s \alpha}+e_{i_{s \beta}} i_{s \beta}\right)\right]+K_{i R_{s}} \int_{0}^{t}\left[-\left(e_{i_{s \alpha}} i_{s \alpha}+e_{i_{s \beta}} i_{s \beta}\right)\right] d t
\end{aligned}
$$

\section{Simulation of direct torque control system based on MRAS}

\section{structure block diagram of the system}

The structure block diagram of direct torque control system for permanent magnet synchronous motor is shown in figure 7 .

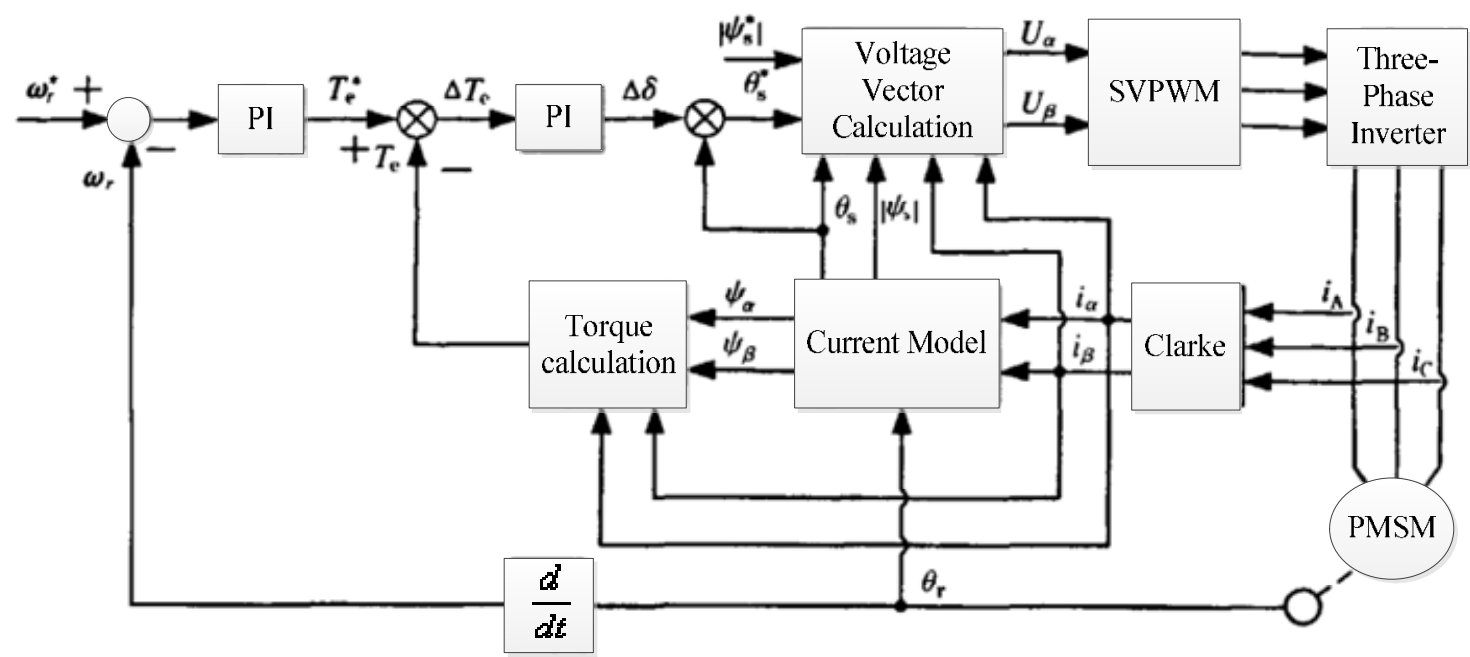

Figure 7 The structure block diagram of direct torque control system for permanent magnet synchronous motor

\section{Simulation}

Using Matlab/simulink simulation platform, a simulation model of the non-speed-sensor direct torque control based on the full-order adaptive state observer MRAS is built. With reference to the previous analysis, add the full-order MRAS model to the direct torque control system. When the torque is fixed, at the time of [0-0.5-1]s, the speed step condition is [1000-100-500] $\mathrm{r} / \mathrm{min}$.

The simulation waveform is shown in figure 8. Figure (a), (b) are the actual speed curve and the identification speed curve, (c) is the estimation error curve.

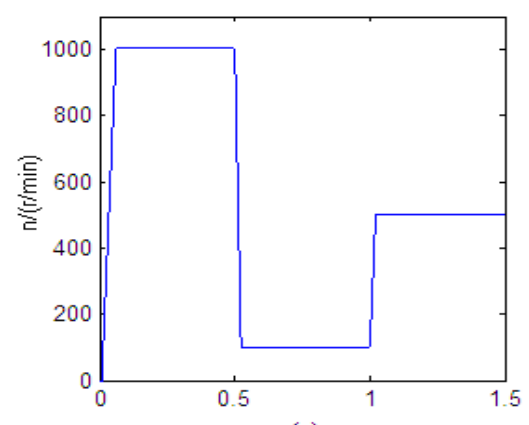

(a)

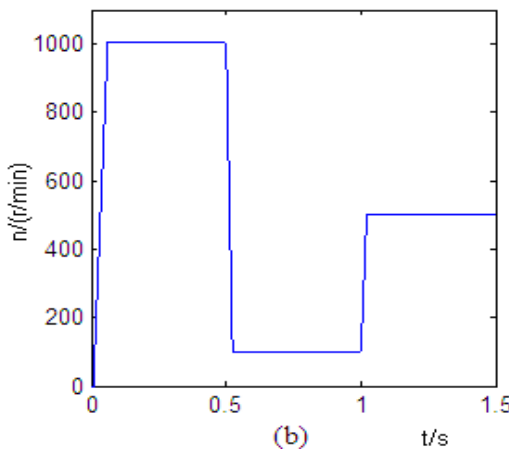

(b)

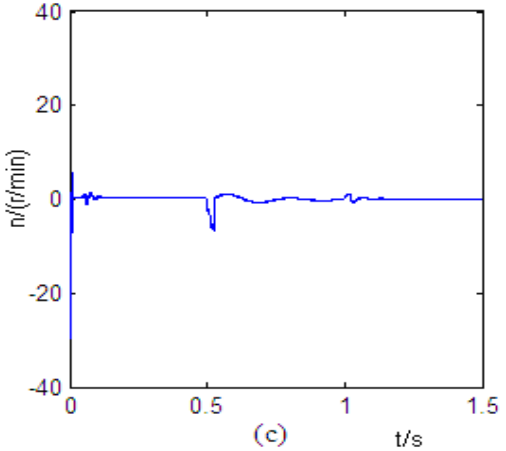

(c)

Figure 8 speed identification of full-order MRAS 
In order to verify the application effect at low speed of the full-order MRAS on DTC, a simulation platform was built at given speed is $80 \mathrm{r} / \mathrm{min}$ and given torque is $10 \mathrm{~N}$.m. Figure $9 \mathrm{~s}$ the simulation results of low-speed state, (a) is the track of the stator magnetic chain, (b) is the current waveform of electron a, (c) is the torque wavdform, (d) the rotating speed waveform.

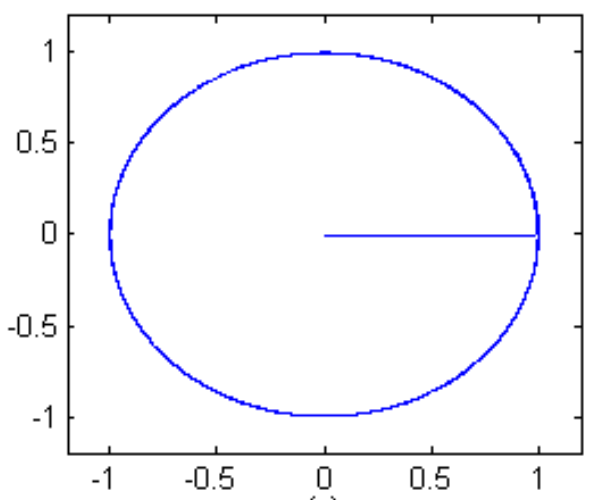

(a)

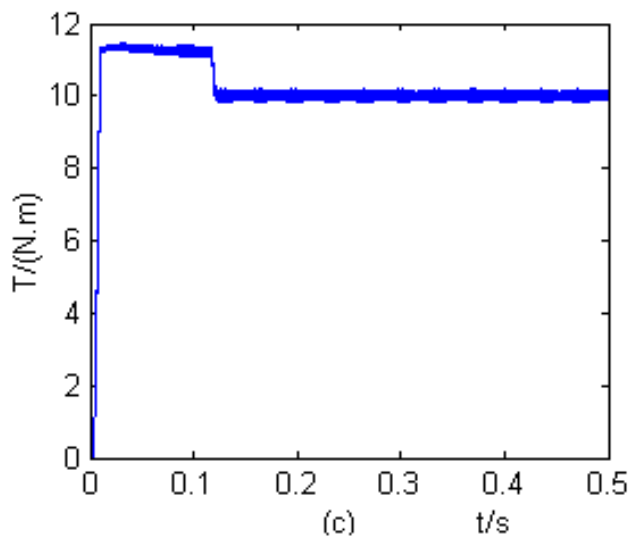

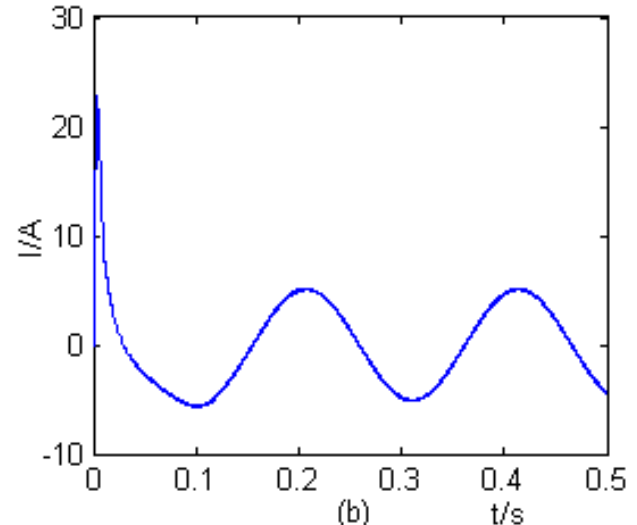

(b)

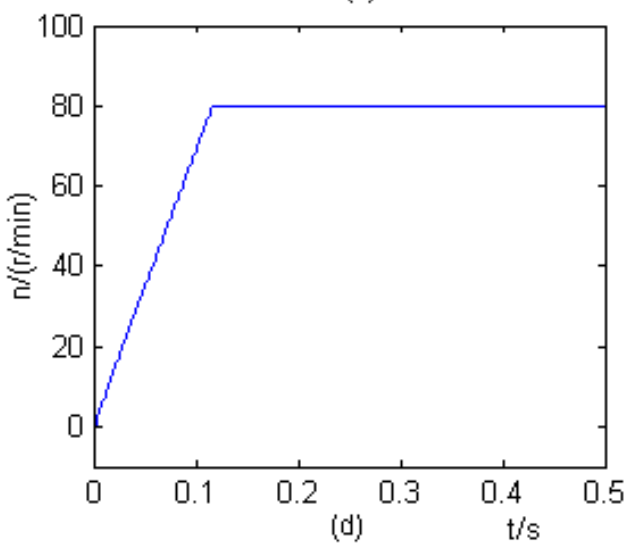

Figure 9 low-speed simulation waveform

Figure 10 shows the system simulation waveform of the load torque step. Given the speed of $500 \mathrm{r} / \mathrm{min}$, the load torque is changed from $10 \mathrm{~N} . \mathrm{m}$ to $5 \mathrm{~N} . \mathrm{m}$ when the time is $0.3 \mathrm{~s}$. FIG. (a), FIG. (b) are the stator flux simulation waveforms of the SVPWM-DTC under the conventional DTC and the full-order self-adaptive state observer MRAS. FIG. (c), FIG. (d) are the torque simulation waveform of two control systems, FIG. (e), figure (f) are the stator current simulation waveform of two control systems. In the same condition, the stator flux linkage path close to the circular under the full-order adaptive state observer MRAS, the distortion of stator flux and stator current is small and the torque ripple is suppressed effectively . 


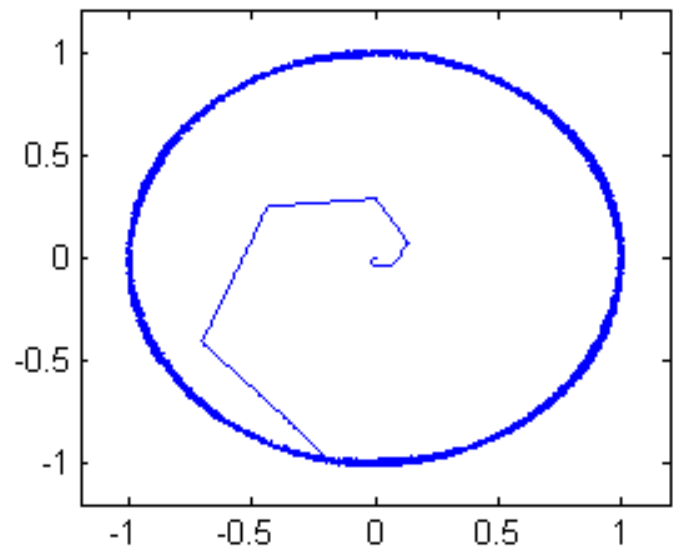

(a)

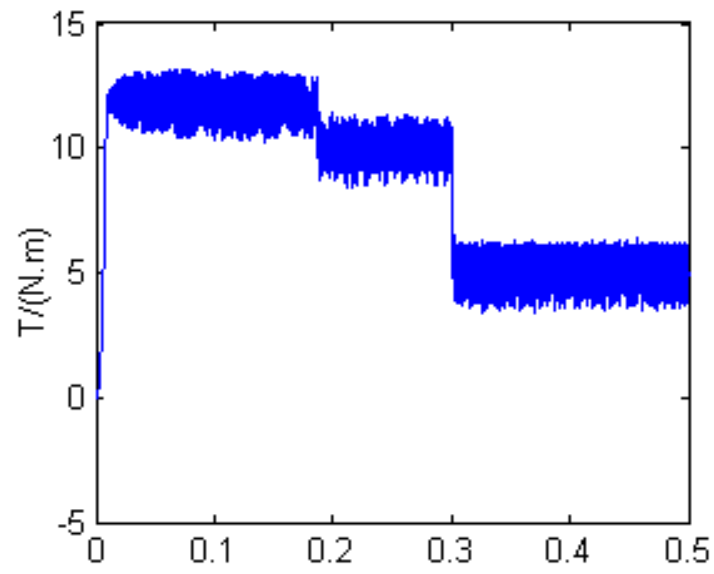

(c)

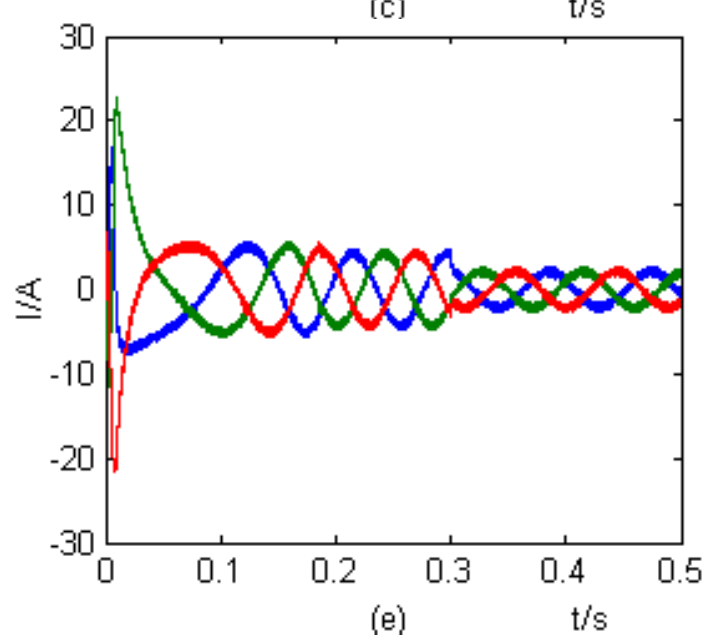

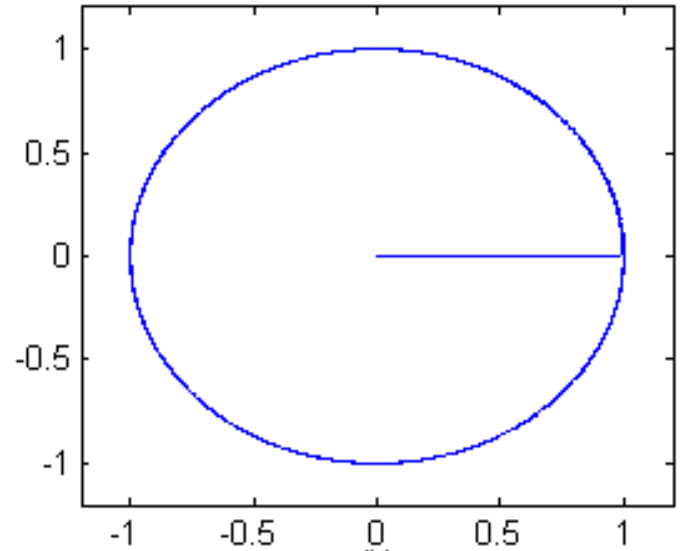

(b)

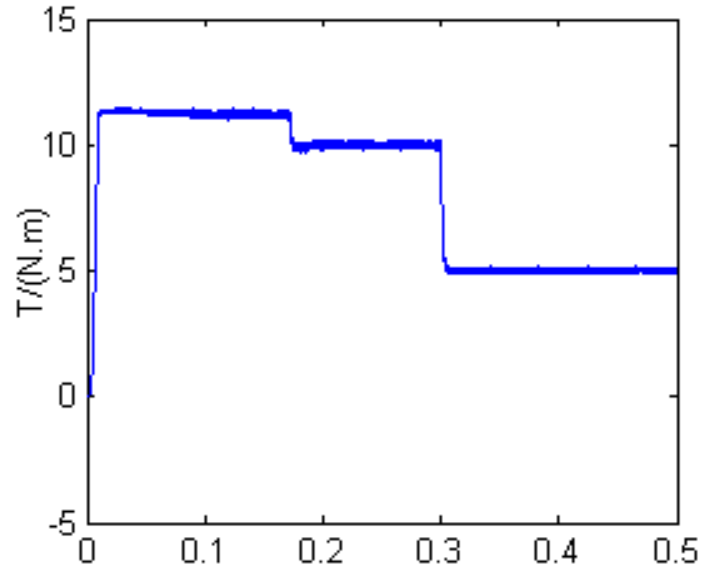

(d)

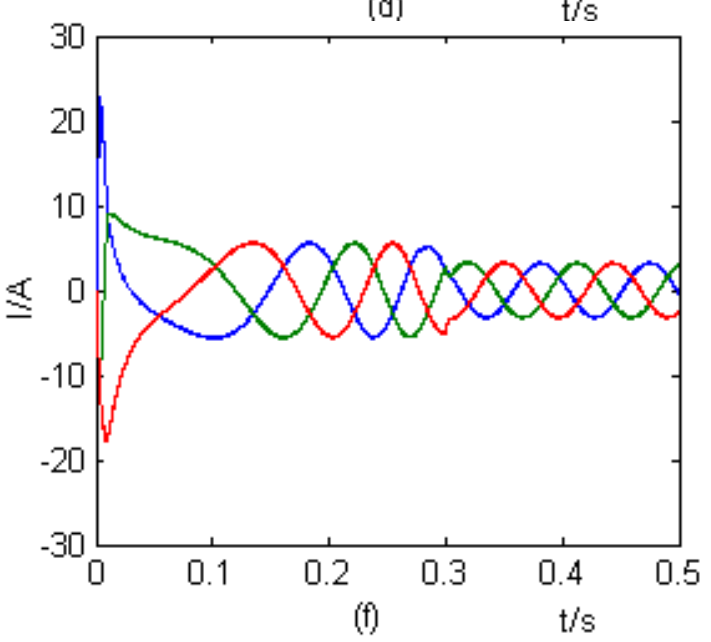

Figure 10 comparison of the simulation waveform of the torque step

\section{conclusion}

Based on the characteristics of tank armored vehicles, this paper establishes a direct torque control strategy for the permanent magnet synchronous motor with no speed sensor. This strategy adopts new speed identification scheme of full-order adaptive state observer, Popov hyperstability theory is used to establish the adaptive law of speed and stator resistance, which can identify speed and stator resistance simultaneously, and return the stator resistance feedback loop to the system, so as to reduce the influence of the change of stator resistance parameters on the speed identification. The simulation results show that this new type of no-speed sensor control strategy is simple and effective, and is a method worthy of further study. 


\section{References}

[1] Depenbrock M. Direct self-control (DSC) of inverter-fed induction machine[J]. IEEE Trans. on Power Electronics, 1988,4: 420-429.

[2] Colin S. Adaptive speed identification for vector control of induction motor without rotational transducers. IEEE Trans. on Industry Applications, 1992, 28(5): 1054 1061

[3] Nik R.N.I., Adbul H.M.Y. An improved stator flux estimation in steady-state operation for direct torque control of induction machines. IEEE Trans. on Industry Applications, 2002, 38(1): $110 \sim 116$.

[4] Liu X.J., Felipe L.R., and Chan C.W. Model reference adaptive control based on neuro fuzzy networks. IEEE Trans. on Systems, 2004, 34(3): 302-309

[5] Kubota H., Matsue K. DSP-based speed adaptive flux observer of induction motor. IEEE Trans. on Industry Applications, 1993, 29(2): 344-348[6] P.G. Clem, M. Rodriguez, J.A. Voigt and C.S. Ashley, U.S. Patent 6,231,666. (2001)

[6] Chen F., Dunnigan MG.. Comparative study of a sliding-mode observer and Kalman filters for full state estimation in an induction machine. IEEE Proceedings Electric Power Applications, 2002,149(1):53-64 